\title{
Classification of Mammograms using Support Vector Machine
}

\author{
Dharmesh Singh and Mandeep Singh \\ ME Student EIED \\ Thapar University, Patiala, Punjab, India \\ Asst Prof EIED \\ Thapar University,Patiala, Punjab, India \\ dharmeshsingh03@gmail.com,mdsingh@thapar.edu
}

\begin{abstract}
In the present work, a computer aided classification system has been proposed for classification of mammogram images into normal, benign and cancer classes. The work has been carried out on thirty Digital Database for Screeningmammography(DDSM) cases consisting of 10 normal, 10 benign and 10 cancer images. The regions of interest (ROI) have been extracted from the right Medio Lateral Oblique (RMLO) part of the mammogram. We extracted $256 \times 256$ pixel size ROI from each case. Texture descriptors based on gray level co-occurrence method by varying the value of inter pixel distance ' $d$ ' from 1 to 8 have been used. The SVM classifier has been used for the classification task. The result of the study indicates that GLCM mean and range features computed at $d=1$ yield the maximum overall classification accuracy of $75 \%$ and $65 \%$ respectively.
\end{abstract}

Keywords: DDSM images, Gray level co-occurrence matrix and Support vector machine

\section{Introduction}

Breast cancer is considered as a most rapidly increased cancer among women in western countries and all the developed cities in India. The American Cancer Society [1] estimates that approximately 230,480 women in the US will be diagnosed with breast cancer, and about 39,520 women will die from breast cancer. A recent report by National Cancer Registry Programs tell the "Breast cancer accounts for $28-35 \%$ of all cancers among women in major cities( Delhi, Mumbai, Ahmedabad ,Chennai etc.) and it is increasing rapidly in large figures". Mammography, biopsy and biopsy needle, these three methods generally used to detect breast cancer. The first step is mammography for detection of breast cancer [3]. A mammogram is an X-ray system to check the breast .Xray mammography is standard procedure for diagnosis breast cancer. The diagnosis result of mammogram is classified into three categories: Normal, benign and cancer. Normal represents mammogram without any cancerous cell, benign represents mammogram showing a tumor but not produced by cancerous cell and cancer represents tumor produced by cancerous cell. It is difficult task to distinguish between among all three categories. Recent use of textural models and machine learning classifiers has established a new research direction todetect breast cancer. Many researcher in the past have used a specific ROI for texture analysis [4-5].

ROI in mammogram image is segmented into maximum possible number of nonoverlapping small squared shape region of fixed size to acquire a large dataset for the further studies. A typical mammogram classification system generally consists of three sequential steps: (1) Extraction of region of interest, (2) features extraction from selected ROI, and (3) classification of mammogram based on extracted features 


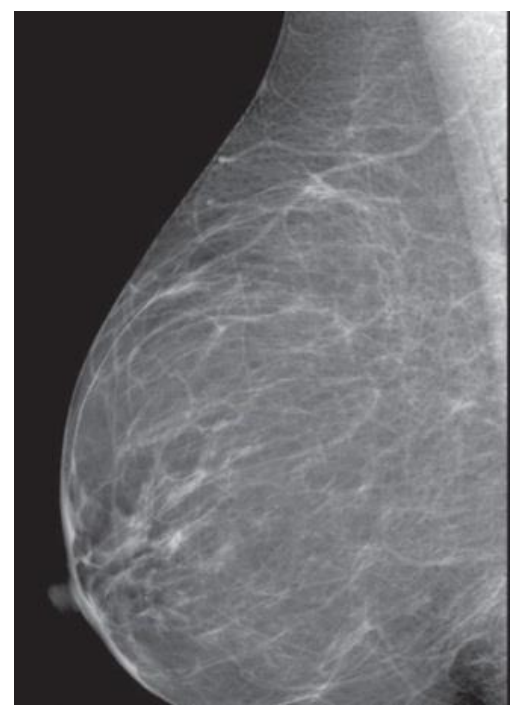

\section{Figure 1.(a) Normal MammogramNote: RMLO Projection}

In this paper, the accuracy of classification problem differentiates between normal, benign and cancer cells using texture descriptors. For achieving this object, textures features using Haralick's gray level co-occurrence matrix (GLCM) [6].are extracted from every ROI from every mammogram case. For classification purpose support vector machine is used.

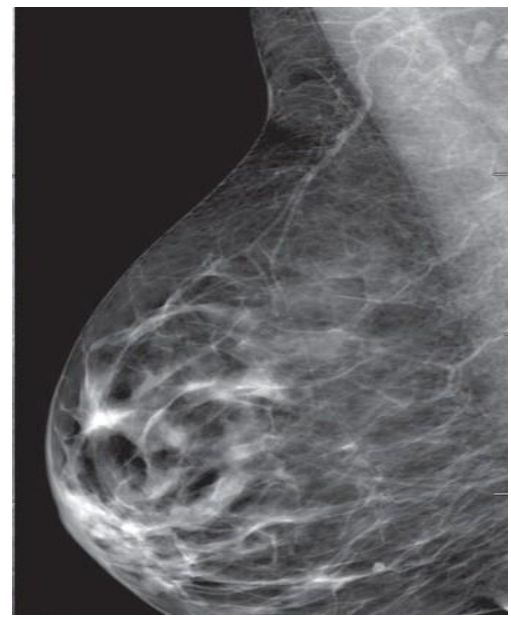

Figure 1.(b) Benign Mammogram 


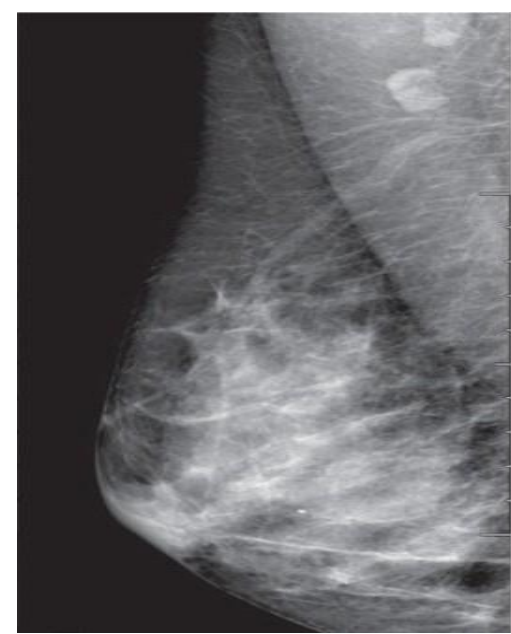

\section{Figure 1.(c) Cancer Mammogram.Note: All Images are Right MLO Projected}

Few studies have been reported in the literature with regard to the classification of DDSMimages. Brief description of their study has been given in Table 1.

Table 1. Literature Review.

\begin{tabular}{|c|c|c|c|c|c|c|}
\hline Author & Image classes & SBT/ROI & $\begin{array}{l}\text { No. of } \\
\text { Images }\end{array}$ & Features & Classifiers & $\begin{array}{l}\text { Acc. } \\
(\%)\end{array}$ \\
\hline $\begin{array}{l}\text { Karssemeijer } \\
\text { et al. [7] }\end{array}$ & 4-Class & SBT & 615 & $\begin{array}{l}\text { Hough } \\
\text { Transform } \\
\text { statistical }\end{array}$ & KNN & 65 \\
\hline $\begin{array}{l}\text { Petroudi et al. } \\
\text { [8] }\end{array}$ & 2-Class & SBT & 132 & $\begin{array}{l}\text { \&gray } \\
\text { level based } \\
\text { features }\end{array}$ & $\mathrm{KNN}$ & $75.7:$ \\
\hline $\begin{array}{l}\text { Oliver et al. } \\
\text { [9] }\end{array}$ & 4-Class & SBT & 300 & $\begin{array}{l}\text { Relative } \\
\text { areas, } \\
\text { center of } \\
\text { mass }\end{array}$ & $\begin{array}{l}\mathrm{KNN} \text {, } \\
\text { Decision } \\
\text { tree }\end{array}$ & 47 \\
\hline $\begin{array}{l}\text { Bovis et al. } \\
{[10]}\end{array}$ & 4-Class & SBT & 377 & $\begin{array}{l}\text { SGLD } \\
\text { Features }\end{array}$ & ANN & 71.41 \\
\hline $\begin{array}{l}\text { Mustra et al. } \\
\text { [11] }\end{array}$ & 4-Class & ROI & 144 & $\begin{array}{l}\text { GLCM } \\
\text { features }\end{array}$ & $\mathrm{KNN}(\mathrm{k}=1)$ & 79 \\
\hline $\begin{array}{l}\text { Kumar, et al. } \\
\text { [12] }\end{array}$ & 4-Class & $\begin{array}{l}128 \times 128 \\
\text { ROI }\end{array}$ & 480 & $\begin{array}{l}\text { Wavelet } \\
\text { packet } \\
\text { texture } \\
\text { descriptors }\end{array}$ & SVM & 73.7 \\
\hline Qu et al. [13] & 2-Class & SBT & 322 & - & E-FELM & 72.6 \\
\hline Z.Chen. [14] & 4 Class & SBT & 322 & $\begin{array}{l}\text { Texton } \\
\text { Features }\end{array}$ & $\begin{array}{l}\text { KNN, } \\
\text { Bayesian }\end{array}$ & 75 \\
\hline Present study & 3-Class & $256 \times 256$ & 120 & $\begin{array}{l}\text { GLCM } \\
\text { range, } \\
\text { GLCM } \\
\text { mean }\end{array}$ & SVM & 75 \\
\hline
\end{tabular}


Note: SBT: Segmented breast tissue. Acc.: Accuracy.KNN: kth nearest neighbor. ANN: Artificial neural network. GLCM: Grey-level co-occurrence matrix. SGLD: Spatial Grey level dependence. SVM: Support vector machine. E-FELM-Evolutionary fuzzy extreme learning machine

The Statistical texture descriptors based on GLCM method have been computed from extracted ROIs by varying the inter-pixel distance $d$ from 1 to 8in two ways viz. $(a)$ GLCM-M (GLCM mean), (b) GLCM-R (GLCM range).

\section{Methodology}

In this paper, a CAD system for classification of mammogram images into normal, benign and cancer classes has been proposed.

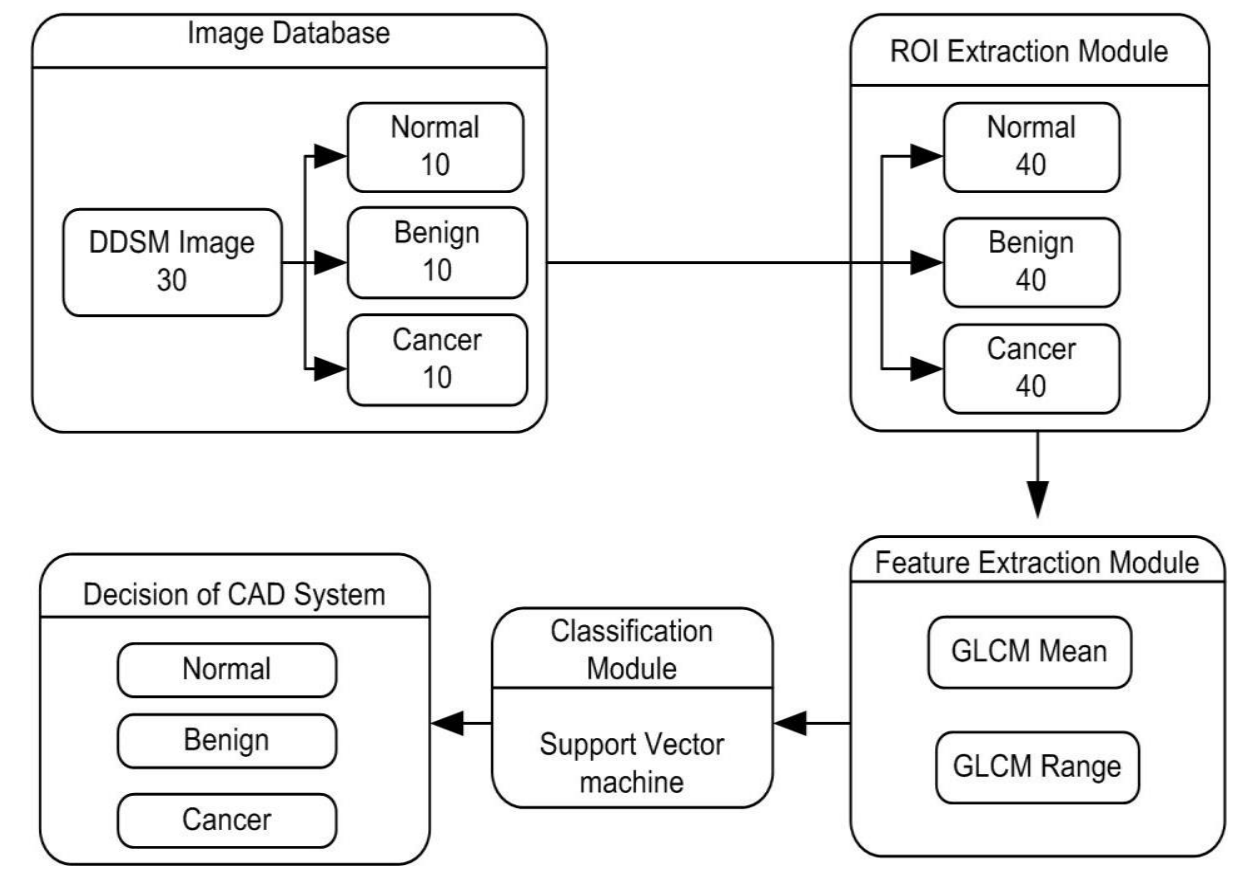

\section{Figure 3. Experimental Workflow}

A. Experimental Workflow

Experimental workflow carried out to design a CAD system is depicted in Figure 3. The CAD system consists of ROI extraction, feature extraction and classification module.

\section{1) Image Database}

In the present work, data set consist of 30 DDSM mammogram images, that include 10 normal, 10 benign and 10 cancer images. Size of each image is $3500 \times 3500$ pixels with 16 bit grey levels and 43.5 microns sampling rate.These are direct digital images, which are used for analysis. Either the left or right MLO projection, we choose R-MLO for this study.

\section{2) ROI Extraction Module}

In ROI extraction module, from eachDDSMimage,120multiple ROIs of size $256 \times 256$ are extracted. ROIs have been taken from random region in case of normal, dense tissue in case of benign, and very dense region of mammogram in case of cancer as shown in Figure 4.. 
In this study, ROIs are manually extracted from DDSM images. ROI size of $256 \times 256$ pixels has been used to extract maximum non-overlapping ROIs. 40 ROIs from 10 normal images, 40 benign ROIs from 10 benign images, and 40 ROIs from 10 cancer images

\section{Feature extraction module}

In this work, exhaustive experimentation has been carried out on 120 ROIs by using statistical second order a) GLCM-M and b) GLCM-R features for different value of $d$ (1 to 8). Feature extraction module has been shown in Figure 5. To derive the statistical texture features from GLCM [16,17], spatial relationship between two pixels is considered. The GLCM-M tabulates the number oftimes the different combinations of pixel pairs of a specific gray level occur in an image fordifferent distances $d=1,2,3 \ldots 8$ and for various directions $=0^{\circ}, 45^{\circ}, 90^{\circ}, 135^{\circ}$ individually as well. Total 13 GLCM-M features namely angular second moment (ASM), contrast, correlation, inversedifference moment, variance, sum average, sum variance, difference, variance, sum entropy, entropy, difference entropy, information measures of correlation-1 and information measures of correlation-2 are computed from each ROI.

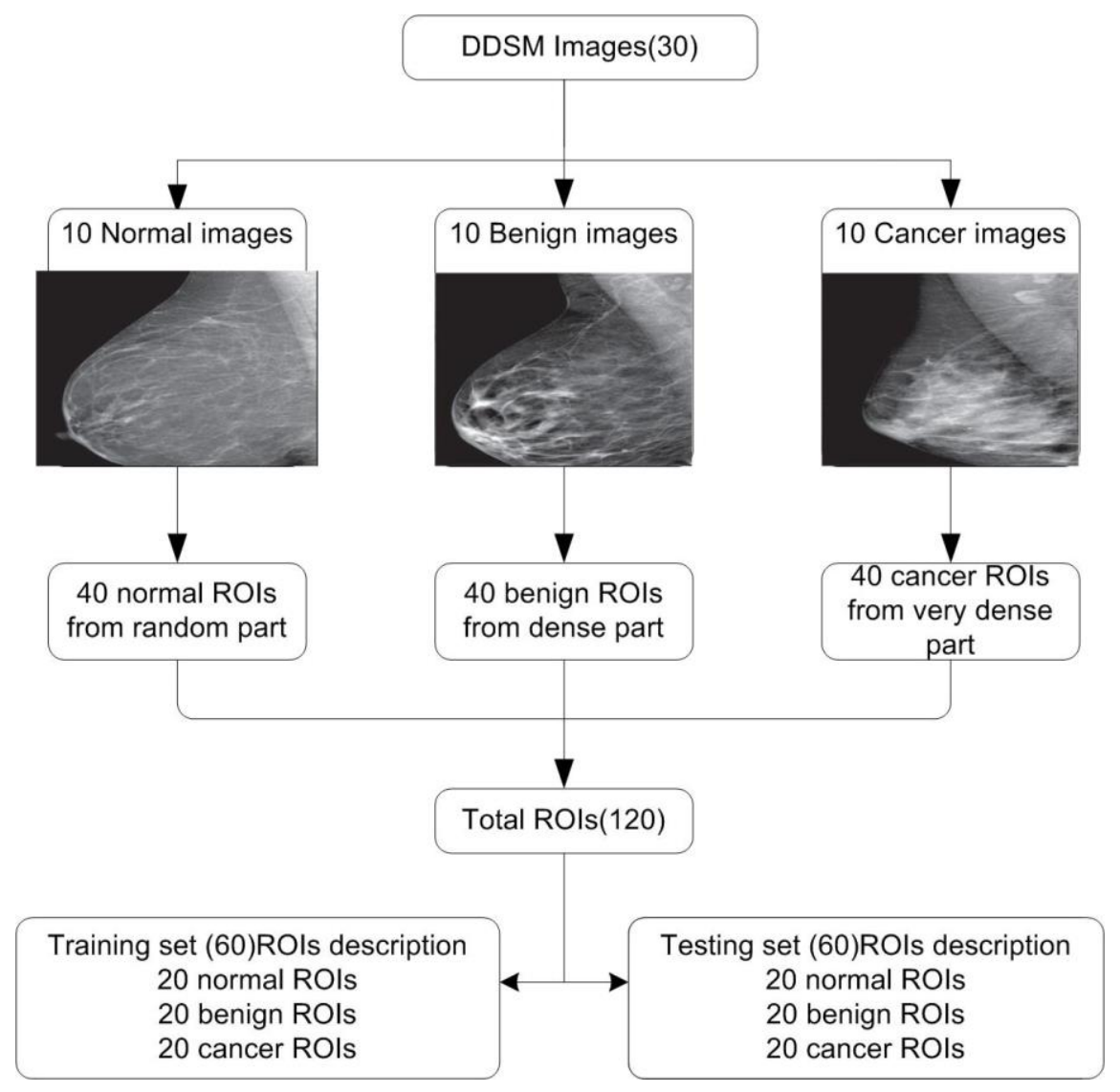

Figure 4. Image Database 


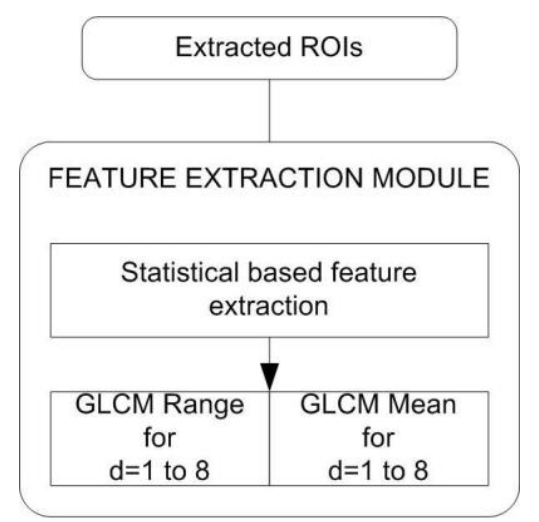

\section{Figure 5. Feature Extraction Module}

These feature values are computed on basis of mean and directions $\left(0^{\circ}, 45^{\circ}, 90^{\circ}\right.$ and $\left.135^{\circ}\right)$. For example, ASM mean (ASM-M) has been computed by thisequation.

$\mathrm{ASM}-\mathrm{M}=\frac{\mathrm{ASM} 0^{\circ}+\mathrm{ASM} 45^{\circ}+\mathrm{ASM} 90^{\circ}+\mathrm{ASM} 135^{\circ}}{4}$

Here, ASM $0^{\circ}$, ASM $45^{\circ}$, ASM $90^{\circ}$, ASM $135^{\circ}$ are angular second moments for mean, calculated at $0^{\circ}, 45^{\circ}, 90^{\circ}$ and $135^{\circ}$ respectively. Similarly 13 GLCM-R features namely angular second moment (ASM), contrast, correlation, inverse difference moment, variance, sum average, sum variance, difference variance, sum entropy, entropy, difference entropy, information measures of correlation-1and information measures of correlation-2 are computed from each ROI for different distances $d$ varying from 1 to 10 . For example, ASM range (ASM-R) has been computed by this equation.

$\mathrm{ASM} \_\max =\max \left(\mathrm{ASM} 0^{\circ}+\mathrm{ASM} 45^{\circ}+\mathrm{ASM} 90^{\circ}+\mathrm{ASM} 135^{\circ}\right)$

$\mathrm{ASM} \_\min =\min \left(\mathrm{ASM} 0^{\circ}+\mathrm{ASM} 45^{\circ}+\mathrm{ASM} 90^{\circ}+\mathrm{ASM} 135^{\circ}\right)$

ASM-R = ASM_max - ASM_min

Here, ASM $0^{\circ}$, ASM $45^{\circ}, \overline{A S M ~} 90^{\circ}$, ASM $135^{\circ}$ are angular second moments for range, calculated at $0^{\circ}, 45^{\circ}, 90^{\circ}$ and $135^{\circ}$ respectively and max and min denotes the maximum and minimum values among all the four directions.

\section{3) Classification Module}

\section{SVM Classifier:}

The goal of the SVM classifier is to design a hyper plane that classifies all training vectors in two classes with the maximum margin. The margin is the distance between hyper plane and the closest elements from this hyper plane. In the present work, Gaussian radial basis function kernel is used for performing nonlinear mapping of data from input space to feature space. While the kernel parameter $\gamma$ controls the curvature of the decision boundary, the soft margin constant C of SVM increase the margin with minimum error possible.The one approach for multi-class classification provided in Lib SVM library [19] has been used in the present work. The optimal values are obtained by grid search procedure to train SVM such that $\gamma \in\{2-5,2-4, \ldots, 25\}$ and $C \in\{2-5,2-4, \ldots, 25\}$ using 10 -fold cross-validation on training data.To prevent the dominance among the features of varied ranges, minmax normalization has been used to rescale the feature valuesbetween 0 and 1 [16-19]. 


\section{Results and Discusssion}

In present work, the following experiments have been carried out.

A. Experiment 1: In this experiment, the classification performance of 8 different GLCM mean feature vector (GMFV) and GLCM range feature vector (GRFV) obtained by varying inter-pixel distance ' $d$ ' from 1 to 8 is tested by using SVM classifier. The brief description of the results obtained is given in Table 2. It is observed that maximum overall accuracy of $75 \%$ and $65 \%$ has been obtained with GLCM-M and GLCM-R features computed at $\mathrm{d}=1$ as shown in Table 2.(a).

It is observed from Table 2.(a) that GLCM-M and GLCM-R results in highest accuracy at $' d{ }^{\prime}=1$. Hence, the detailed result of GLCM-M and GLCM-R is given in Table 2.(b).

B. Experiment 2: In this experiment, the results obtained by experiment 1 are used to improve the classification accuracy of DDSM mammograms into 3 classes. The maximum overall accuracy of GLCM-M and GLCM-R is improved by concatenating the two features at ' $d$ ' $=1$. Results are shown in Figure 3.

Hence as depicted in Table 3, OCA improves on concatenating GLCM-M and GLCM-R feature.

Table 2.(a): Classification Rerformance of GLCM-M and GLCM-R for ' $d$ ' Varying From 1 to 10 .

\begin{tabular}{llllll}
\hline FV & 'd' & $\begin{array}{l}\text { OCA } \\
\text { (in } \\
\text { \%) }\end{array}$ & FV & 'd' & $\begin{array}{l}\text { OCA } \\
\text { (in } \\
\text { \%) }\end{array}$ \\
\hline GMFV & 1 & 75 & GRFVS & 1 & 65 \\
GMFV & 2 & 72.5 & GRFVS & 2 & 63.8 \\
GMFV & 3 & 69.1 & GRFVS & 3 & 63.33 \\
GMFV & 4 & 61.67 & GRFVS & 4 & 56.67 \\
GMFV & 5 & 54.16 & GRFVS & 5 & 55.83 \\
GMFV & 6 & 53.33 & GRFVS & 6 & 55.83 \\
GMFV & 7 & 55 & GRFVS & 7 & 54.16 \\
GMFV & 8 & 50 & GRFVS & 8 & 47.5 \\
\hline
\end{tabular}

Note: GMFV: GLCM-M feature vector GRFV:

GLCM-R feature vector.

Table 2.(b): Classification Performance of 8 Different

GLCM Mean Feature Vectors (GMFV) Using SVM Classifier for Three Classes of DDSM Images for $d=1 \ldots 8$

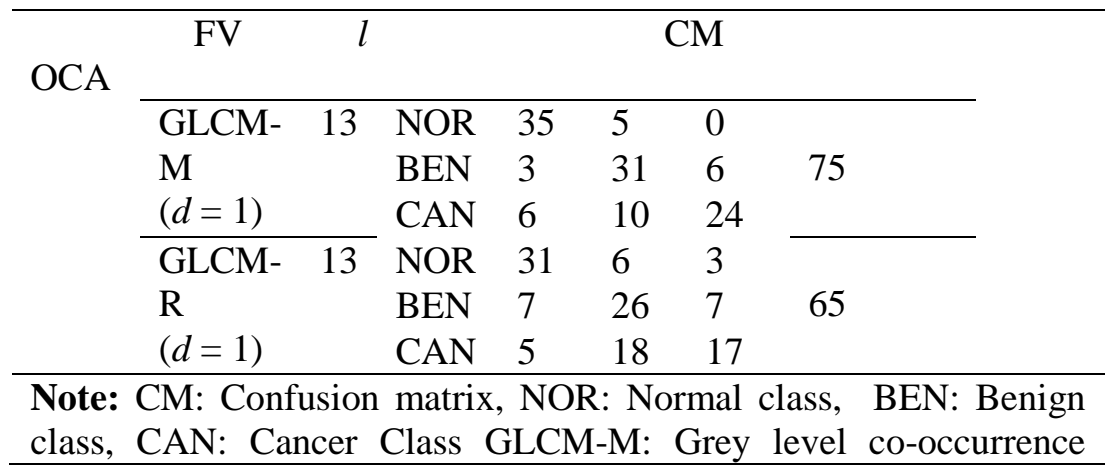


matrix for mean, GLCM-R: Grey level co-occurrence matrix for range, ' $d$ ': Inter pixel distance, TFV: Texture feature vector, $l$ : Length of feature vector, OCA: Overall classification accuracy, FV: Feature vector

\title{
4.Conclusion
}

A study on the classification of DDSM images has been carried out. Texture features such as GLCM-R and GLCM-M are calculated for the classification of normal, benign and cancer DDSM images. The extracted features are classified through SVM classifier. As per the observation of confusion matrix. in all the three experiments, we draw the following inference.

a) GLCM-Mgives the best of $75 \%$ at $d=1$

b) GLCM-R gives the best accuracy of $65 \%$ at $d=1$.

c) Concatenation of GLCM-M and GLCM-R feature gives the maximum accuracy of $77 \%$ at ' $d$ ' $=1$.

Hence, an extensive study using GLCM has been performed on DDSM images of three classes.

Experimental results suggest that best results by classifying the DDSM images into normal, benign and cancer with an accuracy of $77 \%$.

Table 3.: Classification Performance of GLCM-M Concatenated with GLCM-R Using SVM Classifier for Three Classes of DDSM Images for $d=1$

\begin{tabular}{|c|c|c|c|c|c|}
\hline $\mathrm{FV} \quad l$ & $\mathrm{CM}$ & OCA & & & \\
\hline GLCM- 13 & NOR & 60 & 14 & 6 & \\
\hline$M+$ & BEN & 14 & 55 & 11 & 77 \\
\hline $\begin{array}{l}\text { GLCM- } \\
\mathrm{R}(d= \\
1\end{array}$ & CAN & 5 & 5 & 70 & \\
\hline
\end{tabular}

\begin{abstract}
Note: CM: Confusion matrix, NOR: Normal class, BEN: Benign class, CAN: Cancer ClassGLCM-M: Grey level co-occurrence matrix for mean, GLCM-R: Grey level co-occurrence matrix for range, ' $d$ ': Inter pixel distance, TFV: Texture feature vector, $l$ : Length of feature vector, OCA: Overall classification accuracy, FV: Feature vector
\end{abstract}

\section{Acknowledgements}

The authors are thankful to Digital database for screening mammographyresearch laboratory, for providing the mammogram images. The authors are also thankful to Thapar University, Patiala for providing the necessary infrastructure and support.

\section{Reference}

[1] "Cancer facts and figure", http://www.cancer.org/Cancer/BreastCancer/DetailedGuide/breast-cancerkey-statistic (2011)-(2011).

[2] "Population Based Cancer Report", http://ncrpindia.org/Annual_Reports.aspx [Online].

[3] J. Blagojce, K. Ivan, T. Katarina, D. Ivika and L. Suzana, "Mammographic Image Classification Using Texture Features", 9th conference for Informatics and Information Technology, (2012).

[4] H. Sujana, S. Swarnamani and S. Suresh, "Application of artificial neural networks for the classification of liver lesions by image texture parameters", Ultrasound in Medicine \& Biology, vol. 22 no. 9, (1996), pp. pp. 1177- 1181 
[5] M. Tuceryan and A. K. Jain, "Texture Analysis", from The Handbook of Pattern Recognition and Computer Vision, 2nd edition, Editors C.H. Chen, L.F. Pau and P.S.P Wang, World Scientific Publishing Co., (1998), pp 207-248.

[6] R. M. Haralick, K. Shanmugam and I. H. Dinstein, "Textural features for image classification", IEEE Trans Syst Man Cybern, vol. 3, no. 6, pp. 610-621.

[7] N. Karssemeijer, "Automated classification of parenchymal patterns in mammograms", Physics in medicine and Biology, vol. 43, no. 2, (1998), pp.365-389.

[8] S. Petroudi, T. Kadir and M. Brady, "Automatic classification of mammographic parenchymal patterns: a statistical approach", In Proceedings of the $25^{\text {th }}$ Annual International Conference of the IEEE Engineering in Medicine and Biology Society, Cancum, Mexico, vol. 1, (2003), September 17-21, pp. 798-801.

[9] A.Oliver, J. Freixenet and R.Zwiggelaar, "Automatic classification of breast density", In Proceedings of the IEEE International Conference on Image Processing, ICIP, Genova, vol. 2, (2005) September, pp. 1258-1261.

[10] K. Bovis and S. Singh, "Classification of mammographic breast density using a combined classifier paradigm", In medical image understandingand analysis (MIUA) conference, Portsmouth, (C), (2002), pp. $1-4$.

[11] M. G. Mustra and K. Delac, "Breast density classification using multiple features selection", AUTOMATIK, Journal of control measurement, Electronics, Computing and communication, vol. 53, no.4, (2012), pp. 362-372.

[12] I. Kumar, H. S. Bhadauria and J. Virmani, "Wavelet Packet Texture Descriptors based four class BIRADS Breast Tissue Density Classification”,4th International Conference on Eco-friendly Computing and Communication Systems, Procedia, vol.70, (2015), pp.76-84.

[13] Qu Y., Shang.C, Shen Q, “ Evolutionary Fuzzy extreme learning machine for mammographic risk analysis”, International Journal of Fuzzy System, vol. 13, no. 4 ,(2011),pp. 282-291.

[14] Z. Chen, E. Denton and R. Zwiggelaar, "Local feature based mammographic tissue pattern modelling and breast density classification", InProceedings of 4th International Conference on Biomedical engineering and Informatics, Shanghai,; (2011) October 15-17, pp. 351-355.

[15] J. Virmani, V. Kumar, N. Kalra and N. Khandelwal, "Prediction of liver cirrhosis based on multiresolution texturedescriptors from B-mode ultrasound", International Journal ofConvergence Computing, vol. 1, no.1, (2013), pp. 19-37.

[16] J. Virmani, V. Kumar, N. Kalra and N. Khandelwal, "PCA-SVMbased CAD system for focal liver lesions from B-modeultrasound”, Defence Science Journal, vol. 63, no. 5, (2013), pp. 478- 486.

[17] J. Virmani, V. Kumar, N. Kalra and N. Khandelwal, "SVM based characterization of liver cirrhosis by singular value decomposition of GLCM matrix", International Journal of Artificial Intelligence and Soft Computing, vol. 3, (2013), pp. 276-296.

[18] A. Ben-Hur and J. Weston, "A User's guide to support vector machines", Data Min Tech Life Sci, Humana Press:, (2010), pp. 223-239.

[19] Csie.ntu.edu.tw, LIBSVM -- A Library for Support Vector Machines. 
International Journal of Signal Processing, Image Processing and Pattern Recognition Vol. 9, No. 5 (2016) 